\title{
Pesquisas com escalas de crenças sobre abuso e violência sexual: uma revisão integrativa
}

\author{
Surveys with scales of beliefs about abuse and sexual violence: an integrative review
}

Encuestas con escalas de creencias sobre abuso y violencia sexual: una revisión integradora

Fernanda Monteiro Lima ${ }^{1 *}$, Lília lêda Chaves Cavalcante ${ }^{1}$, Daniela Castro dos Reis ${ }^{1}$.

\begin{abstract}
RESUMO
Objetivo: Identificar em artigos científicos publicados quais as escalas utilizadas para conhecer as crenças sobre abuso e violência sexual em pesquisas empíricas. Métodos: Trata-se de uma revisão integrativa da literatura com uso do protocolo PRISMA e adoção de critérios de inclusão que valorizam pesquisas empíricas com escala para mensurar as crenças sobre abuso ou violência sexual. As bases de dados utilizadas foram SciElo, Lilacs, Psycinfo, Scopus e Web of Sciences. As estratégias de busca envolveram descritores em português (escala, questionário, instrumento, crenças, abuso sexual e violência sexual) e em inglês (scale, beliefs, sexual abuse, sexual violence). Resultados: Foram encontrados 128 artigos, porém após a aplicação dos critérios de elegibilidade e exclusão, restaram 8 , sendo 7 escritos em português e 1 em inglês. Considerações finais: As pesquisas encontradas a partir desta revisão sistemática mostraram que os instrumentos utilizados permitiram mensurar as crenças em questão, evidenciando a atualidade daquelas que reforçam a legitimação/tolerância ao abuso e à violência sexual, independentemente da amostra selecionada. $O$ estudo dessas crenças se mostrou relevante na medida em que podem influenciar a produção de conhecimento e mudanças nas relações sociais das quais depende o êxito das políticas públicas voltadas ao enfrentamento do abuso sexual.
\end{abstract}

Palavras-chave: Crenças, Abuso sexual, Violência sexual.

\begin{abstract}
Objective: To identify in published scientific articles which scales are used to know the beliefs about sexual abuse and violence in empirical research. Methods: This is an integrative literature review using the PRISMA protocol and adoption of inclusion criteria that value empirical research with a scale to measure beliefs about sexual abuse or violence. The databases used were SciElo, Lilacs, Psycinfo, Scopus and Web of Sciences. The search strategies involved descriptors in Portuguese (scale, questionnaire, instrument, beliefs, sexual abuse and sexual violence) and in English (scale, beliefs, sexual abuse, sexual violence). Results: 128 articles were found, but after applying the eligibility and exclusion criteria, 8 remained, 7 of which were written in Portuguese and 1 in English. Final considerations: The research found from this systematic review showed that the instruments used allowed the measurement of the beliefs in question, evidencing the relevance of those that reinforce the legitimation/tolerance of abuse and sexual violence, regardless of the selected sample. The study of these beliefs proved to be relevant insofar as they can influence the production of knowledge and changes in social relationships on which the success of public policies aimed at coping with sexual abuse depends.
\end{abstract}

Keywords: Beliefs, Sexual abuse, Sexual violence.

\section{RESUMEN}

Objetivo: Identificar en artículos científicos publicados qué escalas se utilizan para conocer las creencias sobre el abuso y la violencia sexual en la investigación empírica. Métodos: Se trata de una revisión integradora de la literatura utilizando el protocolo PRISMA y la adopción de criterios de inclusión que valoran la investigación empírica con una escala para medir las creencias sobre el abuso o la violencia sexual. Las bases de datos utilizadas fueron SciElo, Lilacs, Psycinfo, Scopus y Web of Sciences. Las estrategias de

${ }^{1}$ Universidade Federal do Pará (UFPA), Belém - PA. *E-mail: fernanda.lima@ntpc.ufpa.br 
búsqueda involucraron descriptores en portugués (escala, cuestionario, instrumento, creencias, abuso sexual y violencia sexual) y en inglés (escala, creencias, abuso sexual, violencia sexual). Resultados: Se encontraron 128 artículos, pero después de aplicar los criterios de elegibilidad y exclusión, quedaron 8, de los cuales 7 estaban escritos en portugués y 1 en inglés. Consideraciones finales: La investigación encontrada a partir de esta revisión sistemática mostró que los instrumentos utilizados permitieron medir las creencias en cuestión, evidenciando la relevancia de aquellas que refuerzan la legitimación/tolerancia al abuso y la violencia sexual, independientemente de las seleccionadas. muestra. El estudio de estas creencias se mostró relevante en la medida en que pueden influir en la producción de conocimientos y cambios en las relaciones sociales de las que depende el éxito de las políticas públicas dirigidas al enfrentamiento del abuso sexual.

Palabras clave: Creencias, Abuso sexual, Violencia sexual.

\section{INTRODUÇÃO}

A violência se mostra como um fenômeno complexo, multifacetado, controverso, perpetrado por indivíduos contra outros indivíduos. Dentro do escopo da violência, encontra-se a violência sexual que é uma de suas formas. A violência sexual alcança todas as faixas etárias, classes sociais e ambos os sexos, atingindo desde recém-natos até idosos. É um fenômeno mundial e está presente tanto em relações heterossexuais quanto homossexuais (SINDEEV A e GUZMÁN-NEGRÓN E, 2018; REIS DC e CAVALCANTE LIC, 2018; REIS DC e CAVALCANTE LIC, 2019).

Devido à magnitude das consequências da violência sexual para a vítima, podendo ser desde lesões físicas, passando pelo risco de adquirirem infecções sexualmente transmissíveis até o desenvolvimento de transtornos psicológicos como, por exemplo, a depressão, a ansiedade, o transtorno de estresse póstraumático. Desse modo, a violência sexual adquiriu progressivamente um caráter endêmico, tornando-se um problema de saúde pública (SINDEEV A e GUZMÁN-NEGRÓN E, 2018; REIS DC e CAVALCANTE LIC, 2018; REIS DC e CAVALCANTE LIC, 2019).

A violência sexual pode ser dividida em abuso sexual e exploração sexual. No abuso sexual, o objetivo é estimular a vítima sexualmente ou obter prazer sexual pessoal. Enquanto na prática da exploração sexual, está presente uma relação mercantil, visando ao lucro, como na prostituição e na pornografia. De acordo com a Organização Mundial de Saúde (OMS), o abuso sexual infantojuvenil é apontado como um dos maiores problemas sociais e de saúde pública na contemporaneidade e que pode estar presente tanto no contexto extrafamiliar como intrafamiliar (OMS, 2014; CEZAR PK, et al., 2017; COSTA LP, et al., 2018).

Diante desse cenário, uma das dificuldades mais evidentes no trato da violência sexual, diz respeito ao desafio de se detectar a amplitude e complexidade desse fenômeno, uma vez que a presença de crenças sociais pode favorecer leituras enviesadas da sua manifestação em diferentes épocas e sociedades, impedindo até mesmo a sua definição e notificação como prevê a legislação e a política pública da área. Assim, o reconhecimento da experiência abusiva depende, por vezes, de crenças sociais que podem promover ou não atitudes capazes de acentuar as dinâmicas traumáticas nos limites da violência sexual (CAMARGO E, 2019; PEREIRA CA, et al., 2019).

Isso porque, crenças podem ser entendidas como uma representação aceita por um grupo, que afeta de forma direta aspectos da cognição, afetividade e do comportamento dos indivíduos, além de refletir na socialização e na formação cultural e ideológica da sociedade. É a partir da construção das crenças sobre indivíduos ou sobre o porquê de eles agirem de determinado modo que o comportamento e as interações sociais são guiados, o que sugere a relevância da produção científica sobre a constituição e configurações (RIBEIRO A e SOEIRO C, 2011; PEREIRA CA, et al., 2019).

Diante do exposto, o objetivo do presente artigo foi identificar em artigos científicos publicados entre os anos de 2000 e 2021, quais têm sido as escalas utilizadas quando se pretende conhecer as crenças sobre abuso e violência sexual presentes em pesquisas empíricas na literatura nacional e internacional, a partir da realização de uma revisão integrativa. 


\section{MÉTODOS}

Esta revisão integrativa foi realizada a partir dos critérios do protocolo Principais Itens para Relatar Revisões Sistemáticas e Meta-análises (PRISMA). Com base nessas orientações, a questão de pesquisa delimitada indagou: quais são os instrumentos utilizados para identificar crenças sociais sobre abuso e violência sexual nas publicações científicas no período de 2000 à 2021. Para tanto, realizou-se uma busca exploratória por artigos nacionais e internacionais sobre o tema utilizando como ferramenta de busca o Portal da Coordenação de Aperfeiçoamento de Pessoal de Nível Superior (CAPES) por este ser mundialmente empregado na divulgação de trabalhos científicos.

Como não foram localizados termos específicos no site dos Descritores em Ciências da Saúde, que apontassem a terminologia comum para esse tipo de pesquisa, a escolha dos descritores se deu a partir da sua evidente associação com tema. Sendo escolhidos seis descritores em português: escala, questionário, instrumento, crenças, abuso sexual e violência sexual e os seguintes termos em inglês: scale, beliefs, sexual abuse, sexual violence, sendo and o termo booleano utilizado na pesquisa. Foram definidas as seguintes bases de dados eletrônicas: Scientific Eletronic Library Online (SciElo), Literatura Latino-Americana e do Caribe em Ciências da Saúde (Lilacs), Psycinfo, Scopus e Web of Sciences.

Desse modo, delimitaram-se os critérios de inclusão e exclusão dos artigos. Critérios de inclusão: artigos completos de livre acesso disponíveis para download; publicados no período de 2000 a 2021. Destaca-se que este recorte temporal foi estabelecido devido a terem sido encontrados textos relevantes enquadrados nesse período na busca preliminar; escritos nas línguas portuguesa, inglesa e espanhola; pesquisas empíricas que utilizem uma ou mais escalas de crenças sobre violência sexual ou abuso sexual; com pelo menos uma das palavras-chave escolhidas para a busca no título ou no resumo.

Critérios de exclusão: artigos que não utilizem nenhuma escala de crenças sobre violência sexual ou abuso sexual; artigos que não apresentam uma das palavras-chave determinadas na busca citadas no título ou no resumo; literatura cinzenta (documentação técnica, anúncios de jornais, relatórios); artigos teóricos e documentais; artigos de revisão sistemática ou integrativa da literatura; livros, teses, dissertações e monografias; artigos escritos em outras línguas que não as especificadas.

A partir da busca por publicações referentes ao tema da presente pesquisa, os artigos encontrados foram enumerados ordinalmente e, em seguida, classificados de acordo com as seguintes categorias: selecionados, duplicados e alheios ao tema. A seleção dos artigos foi feita a partir da leitura do título, bem como do resumo de cada estudo. Nos casos em que os resumos se mostraram insuficientes, foi feita a leitura completa da publicação.

Os artigos selecionados de acordo com os critérios de inclusão foram arquivados em uma planilha eletrônica e os demais, de acordo com os critérios de exclusão, foram descartados. A busca pelos artigos foi realizada pela pesquisadora e compreendeu o período de cinco meses, de setembro de 2021 a janeiro de 2022, com duração média de três horas diárias, três dias por semana. Em seguida, os artigos recuperados foram arquivados.

\section{RESULTADOS E DISCUSSÃO}

Utilizando-se as palavras-chave descritas e os critérios de elegibilidade e de exclusão, foram encontradas 128 produções nas bases de dados conforme são apresentadas a seguir: Lilacs $(n=25)$, SciElo $(n=8)$, Scopus $(n=30)$, Web of Sciences $(n=45)$, Psyclnfo $(n=20)$. Do total dos 128 artigos, 14 foram excluídos por estarem em duplicidade, restando 114 textos. Estes foram analisados sendo excluídos 106 pelos seguintes motivos: 1 tratava-se de uma revisão bibliográfica; 8 por utilizarem entrevista como método, sem a utilização de nenhuma escala; 12 utilizaram escala de crenças, porém sem estar relacionada com abuso sexual ou violência sexual; 38 utilizaram escalas de violência sexual, porém não relacionadas com o tema crenças; e 52 tratavam de um tema alheio, referindo-se ao abuso ou violência sexual e/ou crenças de forma tangenciada. Após essa análise, restaram 8 artigos que cumpriram todos os critérios, sendo 7 escritos em português e 1 em língua inglesa. 
Figura 1 - Fluxograma do percurso metodológico da seleção dos artigos.

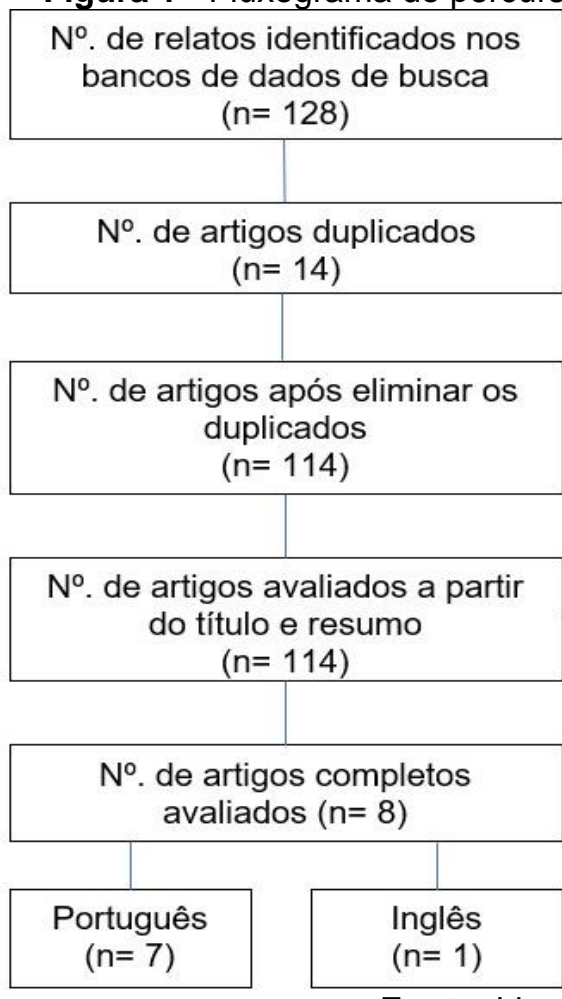

106 artigos que não estavam de acordo com os critérios de elegibilidade.

Fonte: Lima FM, et al., 2022.

O Quadro 1 apresenta a identificação dos oito artigos selecionados a partir da utilização dos critérios de elegibilidade e exclusão nas bases de dados escolhidas com suas respectivas informações que os caracterizam, como: autores do artigo, população estudada, número de participantes, faixa etária e localidade onde foram realizadas as pesquisas. Os artigos estão organizados por ordem alfabética dos nomes dos autores.

Quadro 1 - Caracterização dos artigos selecionados na revisão integrativa.

\begin{tabular}{|c|c|c|c|c|}
\hline Autor(es)/ano & População & $\mathbf{N}^{\circ}$ & Faixa etária & Local \\
\hline $\begin{array}{c}\text { BRINO RF e WILLIAMS } \\
\text { LCA, } 2003\end{array}$ & Educadoras. & 11 & $31-60$ anos & $\begin{array}{l}\text { São Carlos - } \\
\text { SP. }\end{array}$ \\
\hline $\begin{array}{l}\text { FELIZMINO TO e SILVA JP, } \\
2021\end{array}$ & $\begin{array}{l}\text { Estudantes do } 9^{\circ} \text { ano do } \\
\text { ensino fundamental. }\end{array}$ & 284 & 14-18 anos & Aracaju - SE. \\
\hline MARTINS S, et al., 2012 & $\begin{array}{c}\text { Estudantes } \\
\text { universitários. }\end{array}$ & 1000 & 18-24 anos & $\begin{array}{c}\text { Portugal } \\
\text { (Continental). }\end{array}$ \\
\hline PAIVA TT, et al., 2017 & População em geral. & 305 & 18-62 anos & $\begin{array}{l}\text { João Pessoa - } \\
\text { PB. }\end{array}$ \\
\hline PEIXOTO J, et al., 2013 & $\begin{array}{c}\text { Estudantes } \\
\text { universitários atletas e } \\
\text { não atletas. }\end{array}$ & 100 & 18-38 anos & $\begin{array}{c}\text { Portugal } \\
\text { (Região Norte). }\end{array}$ \\
\hline PEREIRA CA, et al., 2019 & $\begin{array}{c}\text { Estudantes } \\
\text { universitários. }\end{array}$ & 417 & $16-50$ anos & $\begin{array}{c}\text { João Pessoa - } \\
\text { PB }\end{array}$ \\
\hline PERRIN N, et al., 2019 & $\begin{array}{l}\text { Membros da } \\
\text { comunidade. }\end{array}$ & 415 & $15-46$ anos & $\begin{array}{l}\text { Somália e } \\
\text { Sudão do Sul - } \\
\text { África. }\end{array}$ \\
\hline $\begin{array}{l}\text { VENTURA MCAA, et al., } \\
2013\end{array}$ & $\begin{array}{c}\text { Estudantes de escolas } \\
\text { secundaristas. }\end{array}$ & 682 & 17 anos & $\begin{array}{c}\text { Portugal } \\
\text { (Coimbra). }\end{array}$ \\
\hline
\end{tabular}

Fonte: Lima FM, et al., 2022.

Presentes no Quadro 2 estão as informações referentes aos instrumentos de crenças utilizados por cada pesquisa empírica, além de recursos complementares, quando utilizados. 
Quadro 2 - Instrumentos utilizados por artigo.

\begin{tabular}{|l|l|}
\hline Autor(es)/ano & Instrumento(s) \\
\hline BRINO RF e WILLIAMS LCA, 2003 & $\begin{array}{l}\text { Registro de abuso sexual (RAS); } \\
\text { Questionário sobre conhecimento e crenças a respeito de abuso } \\
\text { sexual. }\end{array}$ \\
\hline FELIZMINO TO e SILVA JP, 2021 & $\begin{array}{l}\text { Questionário de dados pessoais; } \\
\text { Escala de autoindicação de bullying; } \\
\text { Escala de Crenças sobre Abuso Sexual (ECAS). }\end{array}$ \\
\hline MARTINS S, et al., 2012 & Escala de Crenças sobre Violência Sexual (ECVS). \\
\hline PAIVA TT, et al., 2017 & $\begin{array}{l}\text { Escala de Crenças sobre Violência Conjugal (ECVC); } \\
\text { Escala de autoestima de Rosenberg; } \\
\text { Inventário de Personalidade de Dez Itens (TIPI); } \\
\text { Escala de satisfação com a vida (ESV); } \\
\text { Questionário sociodemográfico. }\end{array}$ \\
\hline PEIXOTO J, et al., 2013 & $\begin{array}{l}\text { Escala de Crenças sobre Violação (ECV); } \\
\text { Sexual Experiences Survey - Short Form Perpetration (SES-SFP). }\end{array}$ \\
\hline PEREIRA CA, et al., 2019 & $\begin{array}{l}\text { Questionário sociodemográfico; } \\
\text { Escala de Crenças sobre Abuso Sexual (ECAS). }\end{array}$ \\
\hline PERRIN N, et al., 2019 & Social Norms and Beliefs about Gender Based Violence Scale. \\
\hline VENTURA MCAA, et al., 2013 & Escala de Crenças sobre Violência Conjugal (ECVC). \\
\hline
\end{tabular}

Fonte: Lima FM, et al., 2022.

Tomando como referência o período selecionado para a presente revisão (2000-2021), o artigo mais antigo foi o das autoras Brino RF e Williams LCA (2003) e o mais recente é de Felizmino TO e Silva JP (2021), ambas realizadas no contexto escolar, sendo a primeira com educadoras e a segunda com estudantes do 9o ano do ensino fundamental. Uma diferença importante entre os dois artigos é que na pesquisa de 2003, as autoras elaboraram um instrumento próprio e fizeram a adaptação de outro, enquanto no artigo de 2021, os autores utilizaram uma escala validada para o contexto brasileiro.

O artigo de 2003 trata-se de um estudo cujo objetivo foi avaliar a eficácia de um programa de intervenção com o intuito de capacitar educadoras para atuar em situações de abuso sexual com seus alunos. No grupo de 11 educadoras, 5 participaram da intervenção e 6 formaram o grupo controle. $O$ estudo foi dividido em duas etapas, a primeira previu o curso de capacitação, semelhante a um workshop, composto por quatro encontros quinzenais. A segunda etapa foi denominada de consultoria, na qual foram realizadas reuniões mensais e individuais com as professoras (BRINO RF e WILLIAMS LCA, 2003).

Utilizados na pesquisa de Brino RF e Williams LCA (2003), antes do primeiro encontro, depois do último encontro da primeira etapa e, novamente, após o término da consultoria, foram aplicados instrumentos para conhecer as crenças sobre o tema e posteriormente para verificar se elas haviam sido alteradas. Quais sejam: 1) Registro sobre Abuso Sexual (RAS) - instrumento traduzido pelas autoras de Hazzard (1984); 2) Questionário sobre Conhecimento de Crenças a Respeito de Abuso Sexual.

Esse segundo instrumento é composto por 43 afirmações de verdadeiro ou falso sobre abuso sexual, elaborado pelas autoras para o estudo. A partir dos resultados, as autoras concluíram que o instrumento RAS mostrou-se adequado para detectar mudanças nos comportamentos das educadoras se for aplicado com intervalos maiores de tempo, o que não foi possível na pesquisa em tela. E quanto ao Questionário, elas pontuaram que as participantes do grupo experimental apresentaram um aumento em seu repertório sobre abuso sexual, enquanto as participantes do grupo controle mantiveram o repertório inalterado (BRINO RF e WILLIAMS LCA, 2003).

Como pode ser verificado no Quadro 1 e no Quadro 2, no cenário da literatura internacional, destaca-se Portugal, com três pesquisas realizadas nesse país, publicadas entre os anos de 2012 e 2013. Enquanto no cenário nacional, destaca-se João Pessoa-PB, com duas pesquisas realizadas nos anos de 2017 e 2019 . Os três estudos elaborados em Portugal utilizam instrumentos diferentes entre si. Cada pesquisa aplicou as seguintes escalas de crenças: Escala de Crenças sobre Violência Sexual (ECVS); Escala de Crenças sobre Violação (ECV) e Escala de Crenças sobre Violência Conjugal (ECVC) (MARTINS S, et al., 2012; PEIXOTO J, et al., 2013; VENTURA MCAA, et al., 2013). 
A pesquisa de Peixoto J, et al. (2013), além de uma escala de crenças, aplicou um instrumento de experiências sexuais, mas não relacionado com crenças, o Sexual Experiences Survey - Short Form Perpetration (SES-SFP). Apesar da diferença entre os instrumentos, as pesquisas apresentaram públicosalvo semelhantes, os estudantes secundaristas e universitários. É importante ressaltar que as escolas secundaristas em Portugal são de cursos profissionais e científico-humanísticos (MARTINS S, et al., 2012; PEIXOTO J, et al., 2013; VENTURA MCAA, et al., 2013).

A pesquisa de Martins S, et al. (2012) teve por objetivo validar um instrumento de investigação sobre violência sexual, adaptado para a população de estudantes universitários portugueses, devido a inexistência de instrumentos com essa capacidade de aferição na época e a relevância do tema, procurando identificar o grau de tolerância/legitimação e conhecendo as crenças que mantém esses comportamentos de violência sexual entre os sujeitos. Os autores concluíram que a escala apresentada possui níveis satisfatórios de precisão e validade, sendo mais um instrumento importante para investigar a tolerância à violência sexual.

O objetivo do estudo de Peixoto J, et al. (2013) foi identificar se estudantes universitários do sexo masculino, praticantes de modalidades esportivas de contato, apresentariam um nível maior de legitimação/tolerância face à violência sexual sobre as mulheres a partir da aplicação da Escala de Crenças sobre Violação (ECV) e se eles apresentariam uma taxa de atos de violência sexual no namoro maior que um grupo de universitários não atletas a partir da aplicação do Sexual Experiences Survey - Short Form Perpetration (SES-SFP).

Os participantes foram divididos em 50 universitários atletas de modalidades de contato e 50 universitários não atletas. Os resultados demonstraram que atletas e não atletas não evidenciaram diferenças estatisticamente relevantes e, de um modo geral, os dois grupos apresentaram um reduzido nível de suporte às crenças que justificam ou desculpabilizam a violência sexual, porém em relação ao comportamento, avaliado pelo segundo instrumento (SES-SFP), os dois grupos apresentaram uma taxa superior de perpetração de atos sexuais abusivos na intimidade no "tipo mais grave", a violação (PEIXOTO J, et al., 2013).

O terceiro estudo realizado em Portugal, apresentado nesta revisão, foi conduzido por Ventura MCAA, et al. (2013) e teve como objetivo identificar crenças e atitudes legitimadoras de violência nas relações de intimidade a partir da utilização da Escala de Crenças sobre Violência Conjugal (ECVC). A população foi composta por 682 estudantes de duas escolas do ensino secundário da região de Coimbra. Os resultados da escala mostraram que, em sua maioria, os estudantes discordam das crenças legitimadoras da violência sexual, porém, os participantes do sexo masculino tiveram uma concordância maior com os comportamentos violentos do que as do sexo feminino.

Assim como o estudo de Ventura MCAA, et al. (2013), a pesquisa de Paiva TT, et al. (2017) também utilizou a Escala de Crenças sobre Violência Conjugal (ECVC), porém com uma população e objetivos diferentes. Em Paiva TT, et al. (2017), o objetivo é relacionar as crenças que legitimam a violência conjugal com os conceitos de autoestima, satisfação com a vida e personalidade. Por isso, além do uso da ECVC, eles aplicaram mais três instrumentos para medir esses conceitos e um questionário sociodemográfico, conforme mostra o Quadro 2.

A amostra selecionada foi formada por 305 pessoas da população geral, maioria mulheres, residentes na cidade de João Pessoa-PB, como pode ser verificado no Quadro 1. Os resultados dessa pesquisa apontaram que fatores pessoais como autoestima e personalidade possuem relação com a legitimação das crenças sobre violência sexual. Porém, a satisfação com a vida não demonstrou essa relação, o que os autores sugerem que possa ser investigado em estudos posteriores (PAIVA TT, et al., 2017).

Os artigos de Felizmino TO e Silva JP (2021) e Pereira CA, et al. (2019) utilizaram a mesma escala, a Escala de Crenças sobre Abuso Sexual (ECAS) que se propõe a apresentar uma medida de tolerância/legitimação do abuso sexual, especificamente cometido contra crianças e adolescentes. A pesquisa de Pereira CA, et al. (2019) teve como objetivo validar para o contexto brasileiro a referida escala, que foi elaborada e validada inicialmente para Portugal, tendo em vista a necessidade de um instrumento que auxilie na obtenção de medidas válidas para o tema. 
A validação se deu em duas fases. A primeira, denominada de "Validação exploratória", participaram 208 estudantes dos cursos de Direito e Psicologia de universidades públicas e privadas, na cidade de João Pessoa-PB, conforme Quadro 1. Além da escala, foi aplicado um questionário sociodemográfico. Na segunda fase, "validação confirmatória", participaram 209 estudantes universitários com as mesmas características da fase anterior, sendo realizados os mesmos procedimentos. Os autores consideraram que a escala apresentou indicadores satisfatórios de validade e consistência interna podendo ser utilizada para o contexto brasileiro.

A pesquisa de Felizmino TO e Silva JP (2021) fez uso da escala validada para o Brasil, com uma amostra de 284 estudantes no 9ำ ano no ensino fundamental, conforme Quadro 1. O objetivo da pesquisa foi identificar se as crenças sobre abuso sexual interferem na autoindicação do bullying. Além dela, foram aplicados um questionário de dados pessoais do aluno e a Escala de Autoindicação de Bullying (Escala B).

Os resultados apontam que o abuso sexual ainda é uma prática cotidiana nas escolas em que a pesquisa ocorreu e, em alguns casos, esse tipo de violência sexual é uma manifestação de bullying. Os autores ressaltam que o tema abuso sexual ainda está atrelado a tabus e inibições o que dificulta o debate e as informações corretas sobre ele. Contudo, os resultados obtidos através da ECAS mostrou que a maioria dos alunos discorda das afirmações que toleram, legitimam e culpabilizam a vítima (FELIZMINO TO e SILVA JP, 2021).

A pesquisa publicada na língua inglesa foi realizada por Perrin N, et al. (2019) com 415 pessoas de comunidades dos países Somália e Sudão do Sul, como mostra o Quadro 1. A pesquisa teve por objetivo o desenvolvimento e o teste de uma escala de Normas e Crenças Sociais sobre a Escala de Violência Baseada no Gênero (VBG) que fosse capaz de medir a mudança ao longo do tempo em normas sociais e crenças pessoais associadas à violência contra mulheres e meninas entre homens e mulheres membros da comunidade em áreas humanitárias complexas e de poucos recursos.

Após a análise dos dados, os pesquisadores concluíram que a escala desenvolvida se mostra como um instrumento com fortes propriedades psicométricas, tendo consistência interna e confiabilidade para medir a mudança em normas sociais prejudiciais associadas à VBG. Sendo, de acordo com os autores, um dos primeiros instrumentos a apresentar boas qualidades para ser aplicado no contexto de áreas humanitárias e poucos recursos (PERRIN N, et al., 2019).

Tendo em vista que a violência sexual é um evento complexo que adquiriu um caráter endêmico, esta torna-se progressivamente um problema de saúde pública, na medida em que deixa sequelas que atingem muito além de questões físicas, refletindo na saúde biopsicossocial das vítimas que podem se tornar mais vulneráveis a desenvolver distúrbios sexuais, quadros de depressão, queixas somáticas, transtorno de estresse pós-traumático, transtornos de ansiedade, alimentares e dissociativos, déficit de atenção e hiperatividade (ANDRADE CS, et al., 2018; PEREIRA CA, et al., 2019).

Assim como podem apresentar alterações comportamentais, cognitivas e afetivas, tais como, sentimento de culpa, diferença em relação aos pares, desconfiança, baixa autoestima, baixo rendimento escolar, conduta hiper sexualizada, abuso de substâncias entorpecentes, fuga do lar, furtos, isolamento social, agressividade, mudança nos padrões de sono, irritabilidade, comportamento autodestrutivos podendo chegar a ideações ou tentativas de suicídio. Os danos aos quais a vítima estará exposta dependem do tempo do abuso (frequência, duração) e do tipo de violência empregada (intensidade), sendo também relevante o tipo de relação da vítima com o autor da agressão e essas repercussões podem se manifestar a curto, médio e longo prazo (ANDRADE CS, et al., 2018; PLATT VB, et al., 2018; PEREIRA CA, et al., 2019).

Neste sentido, os estudos encontrados na presente pesquisa levam em consideração a grande relevância do tema, a lacuna existente na literatura e, principalmente, a importância das crenças para as relações humanas, tendo em vista que elas influenciam na forma como os indivíduos interagem, pois estão diretamente relacionadas aos comportamentos de proteção, prevenção ou violação dos direitos e da integridade humana (PEREIRA CA, et al., 2019; PEREIRA ASR, 2020).

Assim, as escalas elaboradas e utilizadas nos estudos podem demonstrar se determinada amostra apresenta altos níveis de crenças que legitimam/toleram o abuso ou a violência sexual e, a partir do conhecimento desses níveis, é possível elaborar programas de intervenção com o intuito de modificar as 
crenças e, consequentemente, modificar comportamentos que as perpetuam. Colaborando para a elaboração de políticas públicas, além de orientação para profissionais e comunidade em geral, contribuindo para o campo social, científico e de saúde pública (BRINO RF e WILLIAMS LCA, 2003; WALD G, et al., 2018; PEREIRA CA, et al., 2019).

\section{CONSIDERAÇÕES FINAIS}

As pesquisas encontradas a partir desta revisão sistemática da literatura mostraram que os instrumentos utilizados permitiram mensurar as crenças, evidenciando a atualidade daquelas que reforçam a legitimação/tolerância ao abuso e à violência sexual, independentemente da amostra selecionada e do contexto apresentado, demonstrando que as escalas são sensíveis à investigação do objeto de pesquisa. As limitações apontadas pelos autores, em sua maioria, dizem respeito à amostra não ser suficientemente diversificada, destacando a necessidade de mais pesquisas com diferentes público-alvo. Percebe-se que pelo fato de a validação da escala de crenças sobre abuso sexual para o contexto brasileiro ser recente (2019), ainda não foram publicados, nas bases de dados investigadas, muitos estudos com a sua aplicação. O estudo dessas crenças de legitimação/tolerância do abuso e da violência sexual se mostrou relevante na medida em que podem influenciar a produção de conhecimento e mudanças nas relações sociais das quais depende 0 êxito das políticas públicas voltadas ao enfrentamento do abuso sexual.

\section{REFERÊNCIAS}

1. ANDRADE CSS, et al. Notificação da violência física e sexual de crianças e adolescentes: O papel do sistema de vigilância de violências e acidentes/VIVA. Revista Saúde Col. UEFS, Feira de Santana, 2018, 8: 46-53.

2. BRINO RF, WILLIAMS LCA. Capacitação do educador acerca do abuso sexual infantil. Interação em Psicologia, 2003; 7(2): 1-10.

3. CAMARGO E. Gender inequality and intimate partner violence in Bolivia. Revista Colombiana de Sociologia, $2019 ; 42$ (2): 257-277.

4. CEZAR PK, et al. Registros de notificação compulsória de violência envolvendo crianças e adolescentes. Psicologia: Ciência e Profissão, 2017; 37 (2): 432-445.

5. COSTA LP, et al. Autores de agressão sexual em contextos intra e extrafamiliar: Revisão da literatura. MudançasPsicologia da Saúde, 2018; 26(2), 61-69.

6. FELIZMINO TO, SILVA JP. Autoindicação do bullying frente as crenças do abuso sexual. Revista Psicologia, Diversidade e Saúde, 2021; 10(3): 415-428.

7. MARTINS S, et al. Escala de crenças sobre violência sexual (ECVS). Análise Psicológica, 2012; XXX (1-2): $177-191$.

8. ORGANIZAÇÃO MUNDIAL DE SAÚDE (OMS). Relatório mundial sobre a prevenção da violência. 2014. Disponível em: https://nev.prp.usp.br/wp-content/uploads/2015/11/1579-VIP-Main-report-Pt-Br-26-10-2015.pdf. Acessado em: 10 de janeiro de 2022.

9. PAIVA TT, et al. Violência conjugal e suas relações com autoestima, personalidade e satisfação com a vida. Gerais: Revista Interinstitucional de Psicologia, 2017; 10(2): 215-227.

10. PEIXOTO J, et al. Violência sexual no namoro: Os atletas universitários como grupo de risco? Psicologia Edições Colibri, 2013; XXXVII (1): 133-156.

11. PEREIRA ASR. Abuso sexual no gênero masculino: Crenças e mitos (Mestrado em Psicologia Forense) - Instituto de Psicologia e Ciências da Vida. Universidade Lusófona de Humanidades e Tecnologias, Lisboa, 2020; 45 p.

12. PEREIRA CA, et al. Validação da escala de crenças sobre abuso sexual (ECAS) no contexto brasileiro. Psico-USF, 2019; 1: 145-158.

13. PERRIN N, et al. Social norms and beliefs about gender based violence scale: A measure for use with gender based violence prevention programs in low-resource and humanitarian settings. Conflict and Health, 2019; 13:6: 1-12.

14. PLATT VB, et al. Violência sexual contra crianças: Autores, vítimas e consequências. Ciência \& saúde coletiva, 2018; 23 (4): 1019-1031.

15. REIS DC, CAVALCANTE LIC. Autor de agressão sexual de criança/adolescente: Uma caracterização da produção sobre o tema. Ciências \& Cognição, 2018; 23(2): 263-276.

16. REIS DC, CAVALCANTE LIC. Avaliação de distorção cognitiva de autores de agressão sexual de crianças e adolescentes (AASCAS): Revisão sistemática da literatura. Revista da SPAGESP, 2019; 20 (2): 99-116.

17. RIBEIRO A, SOEIRO C. Crenças sobre o abuso sexual em profissionais de educação. VI Congresso Internacional da Sociedade Portuguesa de Psiquiatria e Psicologia da Justiça. 2011.

18. SINDEEV A, GUZMÁN-NEGRÓN E. Perfil epidemiológico, clínico-psiquiátrico y legal del sentenciado por delitos sexuales, establecimiento Penitenciario Lurigancho, Lima. Acta Médica Peruana, 2018; 35(3), 168-173.

19. VENTURA MCAA, et al. Violência nas relações de intimidade: crenças e atitudes de estudantes do ensino secundário. Revista de Enfermagem Referência, 2013; 11: 95-103.

20. WALD G, et al. Modelo comunitario para la promoción, prevención, asistencia y protección ante situaciones de abuso sexual contra niñas, niños y adolescentes. Physis: Revista de saúde coletiva, 2018; 28 (4): 1-25. 